\title{
The influence of a mean magnetic field on three- dimensional magnetohydrodynamic turbulence
}

\author{
By SEAN OUGHTON ${ }^{1}$, ERIC R. PRIEST ${ }^{\mathbf{1}}$ \\ AND WILLIAM H. MATTHAEUS ${ }^{2}$ \\ ${ }^{1}$ Department of Mathematical and Computational Sciences, The University, \\ St Andrews, KY16 9SS, UK \\ ${ }^{2}$ Bartol Research Institute, University of Delaware, Newark, DE 19716, USA
}

(Received 28 February 1994 and in revised form 6 June 1994)

Building on results from two-dimensional magnetohydrodynamic (MHD) turbulence (Shebalin, Matthaeus \& Montgomery 1983), the development of anisotropic states from initially isotropic ones is investigated numerically for fully three-dimensional incompressible MHD turbulence. It is found that when an external d.c. magnetic field $\left(\boldsymbol{B}_{0}\right)$ is imposed on viscous and resistive MHD systems, excitations are preferentially transferred to modes with wavevectors perpendicular to $\boldsymbol{B}_{0}$. The anisotropy increases with increasing mechanical and magnetic Reynolds numbers, and also with increasing wavenumber. The tendency of $\boldsymbol{B}_{0}$ to inhibit development of turbulence is also examined.

\section{Introduction}

Many geo- and astrophysical plasmas may be fruitfully considered within the framework of magnetohydrodynamic (MHD) theory (e.g. Parker 1979). Moreover, it is often the case that these magnetofluids contain, or are threaded by, a large-scale magnetic field $\left(\boldsymbol{B}_{0}\right)$, which varies 'slowly' on both temporal and spatial scales of dynamical interest and importance. Thus, at least locally, $\boldsymbol{B}_{\mathbf{0}}$ may be approximated as being both $(a)$ constant in time, and $(b)$ spatially uniform, approximations which can considerably simplify analysis of the system. For example, various features of the solar corona (e.g. coronal holes with their open field lines, the magnetic structures associated with prominences, and coronal loops and arcades) may be modelled using such a ' magnetofluid $+\boldsymbol{B}_{0}$ ' approximation, albeit crudely in some cases. Other systems where such an approximation is useful include the solar wind, where inertial-range MHDscale fluctuations are subject to an approximate Parker (1958) spiral $\boldsymbol{B}_{0}$, and various fusion devices involving large toroidal magnetic fields (e.g. tokamaks). In this paper we numerically investigate a particularly simple model of such systems, namely a periodic cube of incompressible magnetofluid threaded by a uniform static magnetic field $\boldsymbol{B}_{\mathbf{0}}$.

Of course, the investigation described herein is also of interest to turbulence theorists in its own right, independent of its applicability to any geo- or astrophysical systems. Also, as Shebalin, Matthaeus \& Montgomery (1983, hereafter referred to as SMM) noted, derivation of the Strauss equations (Strauss 1976; Montgomery 1982), which have proved useful in connection with tokamak dynamics, required an initial anisotropy. Thus, if three-dimensional simulations support and/or extend SMM's two-dimensional results regarding the development of anisotropy given a $\boldsymbol{B}_{\mathbf{0}}$, further evidence will have been obtained for the validity of these well-used equations of reduced MHD. We note in passing that the theoretical validity of the Strauss equations 
has recently been put on firmer footing in the limits $\beta \ll 1$ and $\beta \approx 1$, where $\beta$ is the usual ratio of gas and magnetic pressures (Zank \& Matthaeus 1992a).

A decade ago SMM, in a numerical study of freely decaying incompressible twodimensional MHD turbulence, showed that initially isotropic states evolved into strongly anisotropic ones when a uniform external magnetic field was present. Unfortunately, prevailing computational limitations prevented testing of their conjecture that such anisotropy would also be seen in fully three-dimensional systems. Here we report on an extension of this work to three-dimensional systems, and show that their predictions are substantially correct.

By way of review we draw attention to the main results found by SMM. Most of their results were presented in Fourier space, where the wavevector $k$ is the Fouriertransform variable conjugate to the coordinate space variable $\boldsymbol{x}$. We also adopt this approach. SMM found that the anisotropy developed in the direction perpendicular to the applied d.c. field $\left(\boldsymbol{B}_{0}\right)$, in the sense that energy preferentially built up in the modes with wavevectors $\boldsymbol{k}$ perpendicular to $\boldsymbol{B}_{0}$, relative to modes with $\boldsymbol{k}$ parallel to $\boldsymbol{B}_{0}$. A physically appealing explanation for the phenomenon was given based on a weak turbulence analysis of the dynamical equations (i.e. the first-order nonlinear corrections to the solutions of the linearized equations were computed). Briefly, they argued that two excited Fourier modes will resonate effectively with a third, only if the first two modes are oppositely propagating and satisfy certain matching conditions (the nonlinear terms in the MHD equations exactly cancel for Alfvén wave solutions, hence waves propagating in the same direction do not generate additional modes). Numbering these modes 1,2 and 3, respectively, with wavevectors $\boldsymbol{k}_{i}$ and frequencies $\omega\left(\boldsymbol{k}_{i}\right)$, the necessary matching conditions are

$$
\begin{gathered}
k_{3}=k_{1}+k_{2}, \\
\pm \omega\left(k_{3}\right)=\omega\left(k_{1}\right)-\omega\left(k_{2}\right),
\end{gathered}
$$

where modes with frequencies of opposite sign propagate in opposite directions along $\boldsymbol{B}_{0}$. However, since this is a weak turbulence analysis $\omega(\boldsymbol{k})= \pm\left|\boldsymbol{k} \cdot \boldsymbol{B}_{0}\right|$, and the above equations imply that either $\omega\left(\boldsymbol{k}_{1}\right)=0$ or $\omega\left(\boldsymbol{k}_{2}\right)=0$. Thus, either $\boldsymbol{k}_{1}$ or $\boldsymbol{k}_{2}$ is perfectly perpendicular to $\boldsymbol{B}_{0}$. It follows that the third mode can have $\boldsymbol{k}_{\perp}$ greater than that of either of the exciting modes, but $k_{3, \|}$ cannot exceed the maximum of $k_{1, \|}$ and $k_{2, \|}$. Excitations of this nature can therefore readily transfer energy perpendicular to $B_{0}$ in $k$-space, but not parallel to it. The anisotropy was found to increase as each of the following quantities increased: (a) $B_{0}$ (although saturation occurred for $B_{0} \gtrsim 2$ ); (b) wavenumber; $(c)$ Reynolds numbers; and $(d)$ time (although again saturation can occur). As we show below, this behaviour carries through to the fully threedimensional case without essential modification.

Moffatt (1967) has also considered the influence of a uniform magnetic field on MHD turbulence. His analytic study focused on the linear dynamics associated with the early time development of flows where the initial conditions involve a turbulent velocity field but no magnetic fluctuations. The study presented here is therefore complementary to Moffatt's and the relationship will be discussed in more detail in $\S 8$.

The paper is structured as follows. In $\$ 2$ we introduce the equations investigated and give a brief discussion on the numerics. Section 3 presents results from non-dissipative runs, useful for testing the precision and accuracy of the code. Sections 4-6 are the heart of the paper, detailing the development of anisotropy (from initially isotropic states) as a function of mean field strength, Reynolds numbers, and rugged invariants. Section 7 considers the influence of $\boldsymbol{B}_{0}$ on some other aspects of the turbulence, and the paper closes with a discussion section and a summary of the results. 


\section{Definitions, dynamical equations, and numerical details}

The constant-density $(\rho)$ incompressible three-dimensional MHD equations, in the presence of a uniform and static external magnetic field $\left(\boldsymbol{B}_{0}\right)$, may be written in dimensionless form as

$$
\begin{gathered}
\frac{\partial \boldsymbol{v}}{\partial t}+\boldsymbol{v} \cdot \boldsymbol{\nabla v}=-\boldsymbol{\nabla} p^{*}+\boldsymbol{b} \cdot \boldsymbol{\nabla} \boldsymbol{b}+\boldsymbol{B}_{0} \cdot \boldsymbol{\nabla} \boldsymbol{b}+\nu \boldsymbol{\nabla}^{2} \boldsymbol{v}, \\
\frac{\partial b}{\partial t}+\boldsymbol{v} \cdot \boldsymbol{\nabla} \boldsymbol{b}=\boldsymbol{b} \cdot \boldsymbol{\nabla} \boldsymbol{v}+\boldsymbol{B}_{0} \cdot \boldsymbol{\nabla} \boldsymbol{v}+\eta \nabla^{2} \boldsymbol{b}, \\
\boldsymbol{\nabla} \cdot \boldsymbol{v}=0, \\
\boldsymbol{\nabla} \cdot \boldsymbol{b}=0,
\end{gathered}
$$

where $v$ is the velocity field, $b$ the fluctuating magnetic field, and $p^{*}$ is the total (kinetic + magnetic) pressure - determined by the incompressibility condition. The dimensionless kinematic viscosity, $\nu$, and magnetic diffusivity, $\eta$, are both uniform scalars, and their reciprocals respectively define the large-scale mechanical $(R e)$ and magnetic $(R m)$ Reynolds numbers. For convenience we also refer to $\eta$ as the resistivity. Time, $t$, is measured in units of the initial large-scale eddy turnover time, i.e. the unit lengthscale divided by the initial unit velocity scale. Note that since $v$ and $b$ represent fluctuations relative to the mean fields $U(=0)$ and $B_{0}$, they are zero-mean quantities. We take the d.c. magnetic field to be in the $z$-direction, i.e. $B_{0}=B_{0} \hat{z}$. The nondimensionalization is such that for the chosen initial conditions (see below), $B_{0}^{2}$ is the ratio of the energy density associated with $\boldsymbol{B}_{0}$ to that associated with the initial magnetic fluctuations, $b(t=0)$.

The fluid mass density $\rho$ is spatially uniform and constant in time, whereas all other fields are in general functions of space $\boldsymbol{x}=(x, y, z)$ and time $t$. The runs discussed here have approximately zero magnetic helicity at all times (see Stribling, Matthaeus \& Ghosh 1994 $a$ and Stribling, Matthaeus \& Oughton $1994 b$ for a discussion of runs with non-zero $H_{m}$ ) and were performed at unit magnetic Prandtl number (i.e. $v=\eta$ ). Using Braginskii's (1965) forms for the viscosity and magnetic diffusivity of a collisiondominated fully ionized Hydrogen plasma it can be shown (e.g. Hollweg 1985, 1986) that $\nu / \eta \approx 3 \times 10^{-6} T^{4} / n$, where $T$ is the plasma temperature in $\mathrm{K}$ and $n$ the plasma number density $\left(\right.$ per $\left.\mathrm{cm}^{3}\right)$. For geo- and astrophysical conditions this ratio spans an enormous range. In the Sun's photosphere for example it is $\sim 10^{-7}$, while in the corona it is $\sim 10^{10}$. Our computational resources were adequate for examining cases with $v=\eta \approx 1 / 200$, but decreasing either $v$ or $\eta$ by even one order of magnitude requires a code resolution greater than that available to us, in order to maintain accuracy. In this initial investigation the simulations have therefore been restricted to the case $\nu=\eta$.

In these units the electric current density is $j=\boldsymbol{\nabla} \times \boldsymbol{b}$, the fluid vorticity $\boldsymbol{\omega}=\boldsymbol{\nabla} \times \boldsymbol{v}$, and the magnetic vector potential is related to the field by $b=\nabla \times a$, with the stream function defined by $\boldsymbol{v}=\boldsymbol{\nabla} \times \boldsymbol{\psi}$. The Coulomb gauge is assumed to be in effect for both $a$ and $\psi($ e.g. $\nabla \cdot a=0)$. We denote the kinetic and magnetic energies per unit mass by $E^{v}=\left\langle\boldsymbol{v}^{2} / 2\right\rangle$, and $E^{b}=\left\langle\boldsymbol{b}^{2} / 2\right\rangle$, where the angle brackets denote spatial averaging, here assumed to be equivalent to ensemble averaging. We will have occasion to refer to both the bulk values of $E^{v}$ and $E^{b}$ and also to their spectra $E^{v}(k)$, etc. The bulk variables ('globals') characterizing the fluid can be interpreted in two ways: (a) $x$-space averages of the appropriate (scalar) field, e.g. $E^{v}=\left\langle\boldsymbol{v}^{2}\right\rangle / 2$, and (b) integrals of the associated spectra over all wavenumbers, e.g. $E^{v}=\int \mathrm{d}^{3} k E^{v}(\boldsymbol{k})$.

It is well known that the ideal MHD equations support an infinite number of 
invariants. Since invariants place constraints on the evolution and dynamical behaviour of a system it is important to identify the ideal invariants of greatest relevance to real dissipative systems. For turbulent flows these are believed to be the rugged invariants. These quadratic quantities remain invariant even when the ideal MHD equations are truncated at arbitrary maximum and minimum lengthscales (Kraichnan 1967). In contrast, it is believed that the other ideal continuum invariants are destroyed by such a truncation. The three rugged invariants of ideal three-dimensional MHD (with $B_{0}=0$ ) - namely, total energy, cross-helicity, and magnetic helicity - are denoted by $E=E^{v}+E^{b}, H_{c}=\langle\boldsymbol{v} \cdot \boldsymbol{b}\rangle / 2$, and $H_{m}=\langle\boldsymbol{a} \cdot \boldsymbol{b}\rangle / 2$, respectively (see for example, Frisch et al. 1975; Stribling 1991). It is also useful to define the normalized cross-helicity, $\sigma_{c}=2 H_{c} / E$. Again, both the global and the spectral forms of the quantities are useful. Note that when $B_{0} \neq 0$ the magnetic helicity is no longer a rugged invariant (Matthaeus \& Goldstein 1982; Stribling et al. 1994a, b).

Both the spatial and the (discrete) Fourier representations of the fields will be used; for the velocity field these are related by

$$
v(x, t)=\sum_{k} v(k, t) \mathrm{e}^{\mathrm{i} k \cdot x}
$$

where $\boldsymbol{k}$ is the wavevector conjugate to $\boldsymbol{x}$ and having magnitude $k=|\boldsymbol{k}|$. Analogous expansions and definitions hold for the other fields. It will usually be clear from the context whether the spatial or spectral representation of the field is being referred to, so that the field's argument will often be omitted. The time dependence of the fields is also rarely explicitly written.

Equations (3) and (4) (or more specifically their Fourier transforms), with periodic boundary conditions imposed, are solved using a dealiased Fourier Galerkin spectral code (Orszag 1971; Gottlieb \& Orszag 1977; Canuto et al. 1988). In $x$-space the computational domain is a periodic cube of side $2 \pi$ so that when $N$ independent Fourier modes are retained in each spatial direction, the wavevectors are all of the form $\boldsymbol{k}=\left(k_{x}, k_{y}, k_{z}\right)$, with each component being an element of the integer set $\{-N / 2+1, \ldots$, $-1,0,1, \ldots, N / 2\}$. Runs discussed here have $N=32,64$ or 96 . The solenoidal nature of $v$ is enforced via projection of the nonlinear terms in $k$-space. If $b(t=0)$ is solenoidal, then the code maintains this property throughout the run. Dealiasing of the product terms is achieved through use of the ' $\frac{3}{2}$ rule' (Patterson \& Orszag 1971; Canuto et al. 1988). Time integration of the Fourier coefficients is carried out using an explicit second-order Runge-Kutta algorithm. Note that no forcing occurs so that the runs are freely decaying or 'run-down' in nature (the only exceptions are the equilibrium runs discussed in $\S 3$ ).

Initial conditions for the runs are generated in $k$-space. For example, the amplitudes of $v(k)$ are chosen so that the modal kinetic energy spectrum is given by

$$
\begin{aligned}
E^{v}(\boldsymbol{k}) & =\frac{1}{2}|\boldsymbol{v}(\boldsymbol{k})|^{2} \\
& =\frac{C}{1+\left(k / k_{k n e e}\right)^{q}},
\end{aligned}
$$

where $C$ is a normalization constant. Clearly for $k \gg k_{k n e e}$ the spectrum has a powerlaw form $\sim k^{-q}$, while for $k \ll k_{k n e e}$ the spectrum is flat. To determine the phase of each $v(k)$, its real and imaginary parts are assigned using independent Gaussian random variables. Usually only a subset of the retained Fourier modes is initially populated, namely those lying between two limiting values, i.e. $k_{L} \leqslant k \leqslant k_{H}$. Initial conditions for the $b(k)$ are chosen in a similar way, but note that controlling the degree of correlation between $v$ and $b$ allows specification of the cross-helicity. In all cases discussed here the 


\begin{tabular}{|c|c|c|c|c|c|c|c|}
\hline Run & $N$ & $\nu=\eta$ & $B_{0}$ & $\sigma_{c}$ & $t_{\text {final }}$ & $\operatorname{Max} k_{d}(t)$ & Label \\
\hline 1 & 32 & 0.01 & 0 & 0.31 & 4 & 30.5 & Al \\
\hline 2 & . & . & 0.5 & . & & 30.3 & Not \\
\hline 3 & & . & 1.0 & . & . & 29.8 & . \\
\hline 5 & 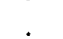 & . & 4.0 & . & $\cdot$ & 28.0 & 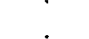 \\
\hline 4 & & - & 8.0 & & & 28.0 & 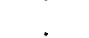 \\
\hline 7 & 32 & 0 & 0 & 0.31 & 15 & - & $\mathrm{A} 2$ \\
\hline 8 & & 0 & 1.0 & & 30 & - & . \\
\hline 9 & 32 & 0.01 & 0 & 0.01 & 4 & 29.3 & B1 \\
\hline 10 & & . & 0.1 & . & . & 29.3 & . \\
\hline 11 & . & . & 0.5 & . & . & 29.0 & . \\
\hline 12 & 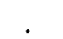 & . & 1.0 & . & . & 28.4 & . \\
\hline 13 & & . & 3.0 & . & . & 26.2 & . \\
\hline 14 & & & 8.0 & . & . & 25.3 & 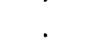 \\
\hline 15 & 32 & 0.005 & 1.0 & . & 4 & 44.1 & B2 \\
\hline 16 & 32 & 0.01 & 1 & -0.03 & 8 & 22.5 & $\mathrm{Cl}$ \\
\hline 17 & 32 & 0.005 & 1 & . & . & 36.2 & \\
\hline c9 & 64 & 0.005 & 0 & . & . & 38.9 & $\mathrm{C} 2$ \\
\hline $\mathrm{cl} 0$ & & . & 0.1 & . & . & 38.8 & . \\
\hline cll & . & 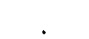 & 1.0 & . & 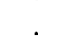 & 36.5 & . \\
\hline $\mathrm{c} 12$ & . & r. & 3.0 & . & . & 32.7 & . \\
\hline c20 & & & 8.0 & & 5 & 32.4 & \\
\hline cl4 & 96 & 0.001 & 1.0 & 0 & 6 & 110 & D1 \\
\hline
\end{tabular}

TABLE 1. Run Parameters. A dot indicates that the value in the column is the same as the last one mentioned in the same column. Extra space between rows sets off runs having distinct initial conditions. For example, all the A runs have the same initial Fourier coefficients. However, the Al and $\mathrm{A} 2$ runs differ in the values of $\nu$ and $\eta$ used. In each case the maximum wavenumber which is resolved in all directions is $k_{\max }=N / 2$

total energy $E$ is initially unity and is equipartitioned between the two forms at all scales, i.e. the Alfvén ratio, $r_{A}(k)=E^{v}(k) / E^{b}(k)$ is unity for each excited mode.

While ideally very large Reynolds number runs should be performed, our computing resources were unfortunately such that only relatively low values of these parameters could be used, and even then most runs are under-resolved. By this we mean that the dissipation wavenumber, $k_{d}$, characterizing the flow is greater than the maximum resolved wavenumber. For the case of unit magnetic Prandtl number, we define $k_{d}$ using

$$
k_{d}^{4}=2 \frac{\left\langle\omega^{2}\right\rangle+\left\langle j^{2}\right\rangle}{\nu^{2}} .
$$

Modes with $k \gg k_{d}$ are heavily damped, and do not contribute significantly to the nonlinear dynamics. However modes with $k \sim k_{d}$ are dynamically significant and must be resolved to achieve accurate simulations (see for example Domaradzki, Rogallo \& Brachet 1993). In practice, if a numerical simulation resolves $k_{d}$, there is a good reason to be confident of the run's accuracy; however, if the reverse holds the accuracy is questionable, since errors at small scales will eventually lead to errors in the large-scale modes. Other consequences of under-resolution, in the context of the anisotropies which develop when $B_{0} \neq 0$, will be discussed in $\S 5$.

Finally in this section we outline the types of runs performed. The procedure followed involved generating several distinct sets of runs. Within each set only one or two parameters were varied; these included $B_{0}$, the Reynolds numbers, spatial resolution, and the cross-helicity. Consequences of varying other parameters, such as 


$\begin{array}{lccrcc}\text { Label } & k_{L} & k_{H} & k_{\text {knee }} & \Omega(t=0) & J(t=0) \\ \text { A1, A2 } & 3 & 8 & 1000 & 15.1 & 15.5 \\ \text { B1, B2 } & 3 & 8 & 1000 & 10.3 & 10.2 \\ \text { C1, C2 } & 1 & 3 & 4 & 2.7 & 2.6 \\ \text { D1 } & 1 & 3 & 4 & 2.1 & 2.1\end{array}$

TABLE 2. Some parameters characterizing the distinct sets of initial Fourier coefficients. All runs had $E^{v}=E^{b}=\frac{1}{2}$ initially

the Alfvén ratio, will be discussed at a later date. This 'all other things being equal' approach, while perhaps somewhat artificial (particularly given the nonlinear nature of the turbulent interactions), does allow the various influences on the development of the anisotropies to be considered as independently as possible. Tables 1 and 2 summarize the parameters of runs referenced in this paper.

\section{Non-dissipative results}

Absolute equilibrium theories of MHD turbulence - that is, the (Gibbsian) statistical mechanics of the truncated and non-dissipative MHD equations in $k$-space-yield quantitative predictions for both the shape and the actual levels of various spectra (Kraichnan 1967; Frisch et al. 1975; Kraichnan \& Montgomery 1980; Stribling \& Matthaeus 1990). These predictions are non-physical since lack of dissipation means that excitations which encounter the high- $k$ 'wall' in wavenumber space bounce back into the retained modes, mostly into the smaller scales because the $k$-space dynamics is approximately local. However, the reflected energy eventually modifies the character of the spectrum at all scales so that absolute equilibrium spectra are generally distinctly different from spectra associated with dissipative turbulence. Despite this Kraichnan (1973) has argued that the absolute equilibrium theories should still provide accurate information regarding spectral transfer directions. The idea is that since within each local wavenumber band the eddy turnover time and the time to relax to absolute equilibrium are of the same order, the fluid should tend to be in approximate absolute equilibrium (locally in $k$-space). Given these quantitative predictions, and assuming that time averaging is equivalent to ensemble averaging (i.e. ergodicity of the system), it follows that simulations with $\nu=\eta=0$ can be used to test code accuracy. We now discuss a few runs of this type.

Stribling (1991) and Stribling \& Matthaeus (1990) have recently shown that when $H_{m}=0$ or $B_{0} \neq 0$, the following formulae for the modal spectra hold:

$$
\begin{gathered}
\left\langle E^{v}(\boldsymbol{k})\right\rangle=\left\langle E^{b}(\boldsymbol{k})\right\rangle=\frac{E}{2 N_{t o t}}, \\
\left\langle\boldsymbol{H}^{c}(\boldsymbol{k})\right\rangle=\sigma_{c}\left\langle E^{v}(\boldsymbol{k})\right\rangle,
\end{gathered}
$$

where $N_{\text {tot }}$ is the number of retained modes, and the angle brackets indicate ensemble averaging. All three spectra are independent of $\boldsymbol{k}$ and thus flat. Note that in this limit $\left(H_{m}=0\right.$ or $B_{0} \neq 0$ ) the absolute equilibrium predictions $(a)$ are independent of $B_{0}$, and (b) do not suggest that partial condensation of $H_{c}$ into the $k_{\text {min }}$ modes occurs (Stribling \& Matthaeus 1990).

Figure 1 displays some spectral results from the non-dissipative run 8 . The spectra shown are obtained by time averaging over the final ten time units of the run, with the absolute equilibrium predictions plotted as dashed lines. Note that before time 

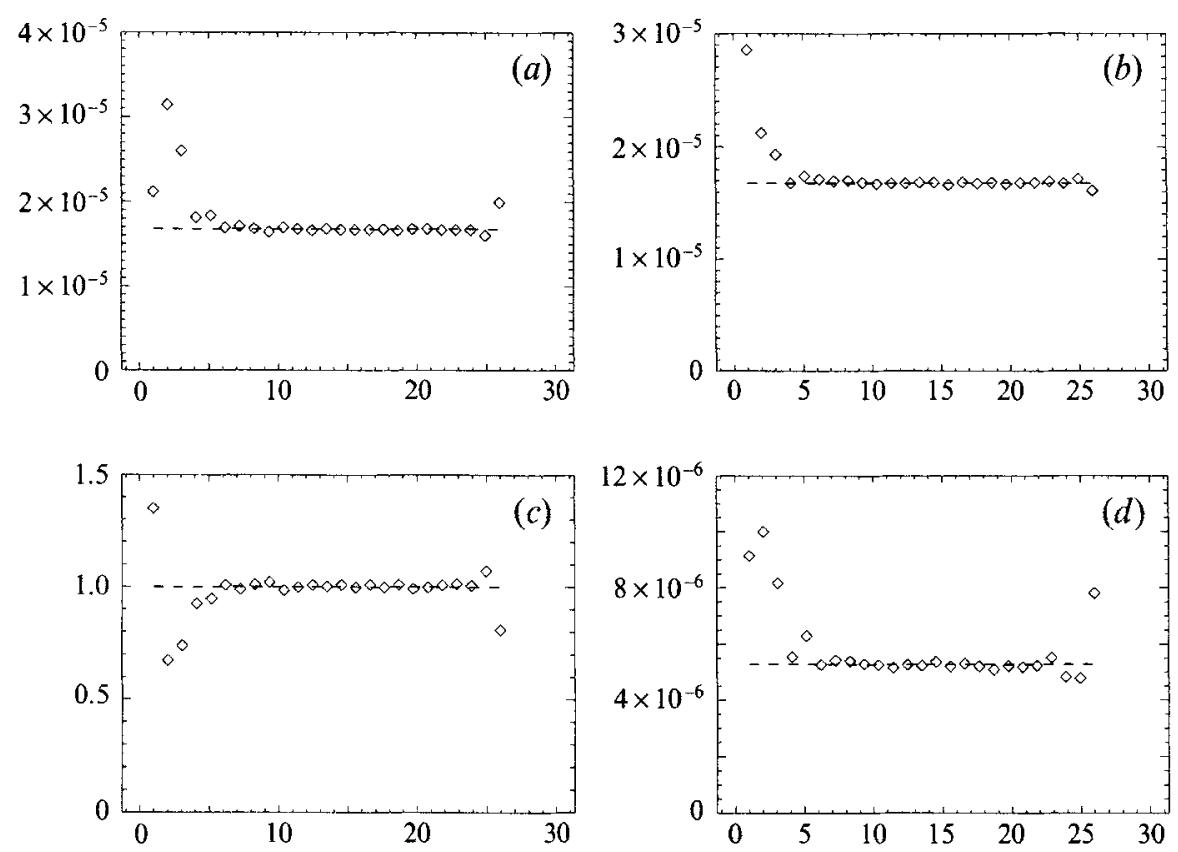

FIGURE 1. Time-averaged omnidirectional spectra for run 8 . The horizontal coordinate is wavenumber. Diamonds are numerical data points and the dashed curves the absolute equilibrium ensemble predictions: $(a)$ magnetic energy, $(b)$ kinetic energy, $(c)$ Alfvén ratio, and $(d)$ cross-helicity.
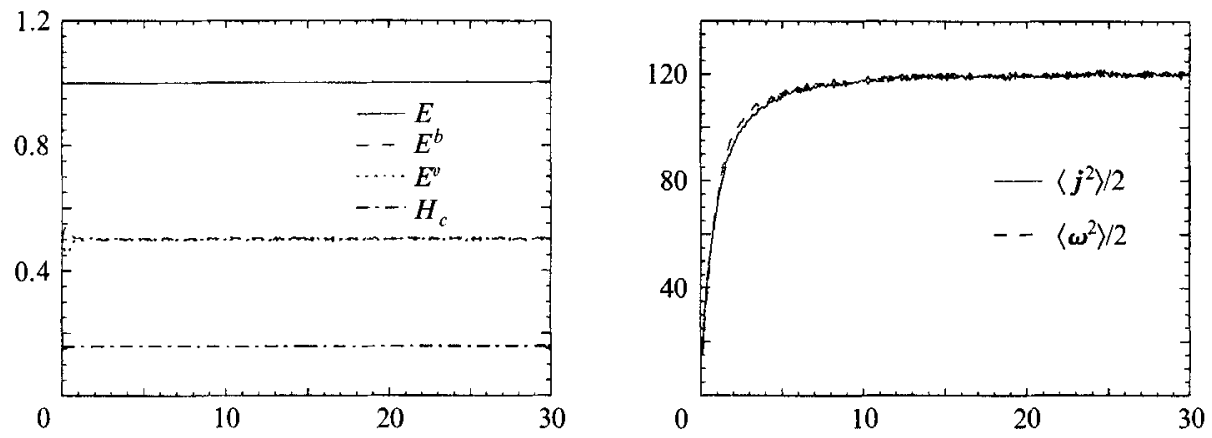

FIGURE 2. Time histories of assorted bulk quantities for run 8 . The horizontal coordinate is time (in units of the initial large-scale eddy turnover time). See $\$ 2$ for symbol definitions.

averaging occurred, the individual modal spectra were directionally averaged; that is, modes with values of $\boldsymbol{k}$ having the same magnitude were averaged over. Clearly, after such averaging modes with values of $k$ having high degeneracy, with respect to $k$, are more likely to give accurate results than those having lower degeneracy. The quantitative agreement between theory and simulation is seen to be excellent at all but the smallest and largest wavenumbers, which is indeed where the degeneracies are low. At low $k$ not many modes exist so the statistics are intrinsically poor, while the finite truncation at high $k$ removes many modes which would otherwise contribute. Similar plots for earlier time intervals show that the high- and low-wavenumber agreement improves as later time intervals are used.

Figure 2 shows time histories of some bulk quantities from the same run. Clearly the non-zero rugged invariants $\left(E\right.$ and $\left.H_{c}\right)$ are accurately conserved. The evolution of the enstrophy, $\Omega=\left\langle\omega^{2}\right\rangle / 2$, and the mean-square current density, $J=\left\langle j^{2}\right\rangle / 2$, are also 
plotted. Their charging-capacitor-like behaviour is in stark contrast to the growthpeak-decay curves characterizing dissipative flows (cf. figure 3). Analogous plots for other non-dissipative runs (not shown) display similarly good agreement with theory; in particular, the time-averaged spectra show no dependence on $B_{0}$. Furthermore, these non-dissipative runs are all isotropic for $t \gtrsim 2$, which may be interpreted as follows. Initially the excitations cascading out in $k$-space are unaware of the high- $k$ 'wall'. Thus, the nonlinear interactions proceed as if a finite dissipation scale exists and the anisotropy with respect to $\boldsymbol{B}_{\mathbf{0}}$ develops. However, once the excitations start being reflected at the 'wall', energy begins to build up at high wavenumbers and the spectral character is modified. After a characteristic time or so the energy has been redistributed such that there is approximate equipartition amongst all the modes, leading to overall isotropy. In contrast, the presence of dissipation leads to anisotropies which persist throughout the runs, as we now discuss.

\section{Development of anisotropy: influence of $B_{0}$}

Having briefly discussed the evolution of non-dissipative MHD turbulence, we now turn to dissipative runs. As is well known, the infinite Reynolds number limits are highly singular - the equations of motion being of higher differential order when $v$ and $\eta$ are non-zero. Hence, behaviour quite different from that of the previous section is to be expected, even for runs with otherwise identical initial conditions and parameters. Indeed, our main result is that a d.c. magnetic field can substantially alter the evolution of MHD turbulence, generating anisotropic fields out of isotropic ones in a few eddy turnover times. Such features are perhaps to be expected since $B_{0}$ imposes a preferred direction; however, it should be noted that in the corresponding non-dissipative runs, the initial development of anisotropy is soon reversed and isotropy restored.

The first set of dissipative results we present are for the C2 runs. As indicated in table 1 these runs have the same initial conditions, resolution, and dissipation coefficients; namely, low cross- and magnetic helicity, unit Alfvén ratio, $N=64$, and $\nu=\eta=1 / 200$. The important distinction between the runs is the value of $B_{0}$, which ranges from 0 to 8 . We will compare these runs using two types of diagnostic quantities: (a) conventional bulk (or global) properties of the flow, and $(b)$ ratios of various mean wavenumbers computed parallel and perpendicular to $\boldsymbol{B}_{\mathbf{0}}$. While the former provide a lumped or gross picture of some of the differences between the runs, such quantities are not really suitable for elucidating or extracting anisotropic features of the flows, prompting introduction of the latter.

A few brief observations regarding time histories of the bulk quantities for the $\mathrm{C} 2$ runs are in order. For clarity, only results from runs $c 9, \mathrm{cl1}$, and $\mathrm{c} 12$ (with $B_{0}=0,1$ and 3) will be displayed in the next two figures. These features will be considered in more detail in $\S 7$, and are mentioned here mainly to give a feel for the runs as a whole. Consequently, no real attempt to explain the features and trends is made at this point.

While qualitatively similar behaviour is observed across runs (figure 3), several trends are evident. First, the energy plots show that increasing $B_{0}$ tends to make $E^{v}$ and $E^{b}$ more nearly equal; that is, it better enforces $r_{A}=1$, in accord with the Alfvén effect (Kraichnan 1965; Pouquet, Frisch \& Léorat 1976). Second, the maxima in the enstrophy $(\Omega)$ and the mean-square current density $(J)$ decrease as $B_{0}$ increases, suggesting that $B_{0}$ acts to suppress or inhibit the turbulence (see $\S 7$ ).

As an aside, we note that the total energy remaining at the end of these (relatively short) runs is $\approx 10-20 \%$ of the initial value, emphasizing that at these low Reynolds numbers dissipation is rather more important (relative to the nonlinear dynamics) than 

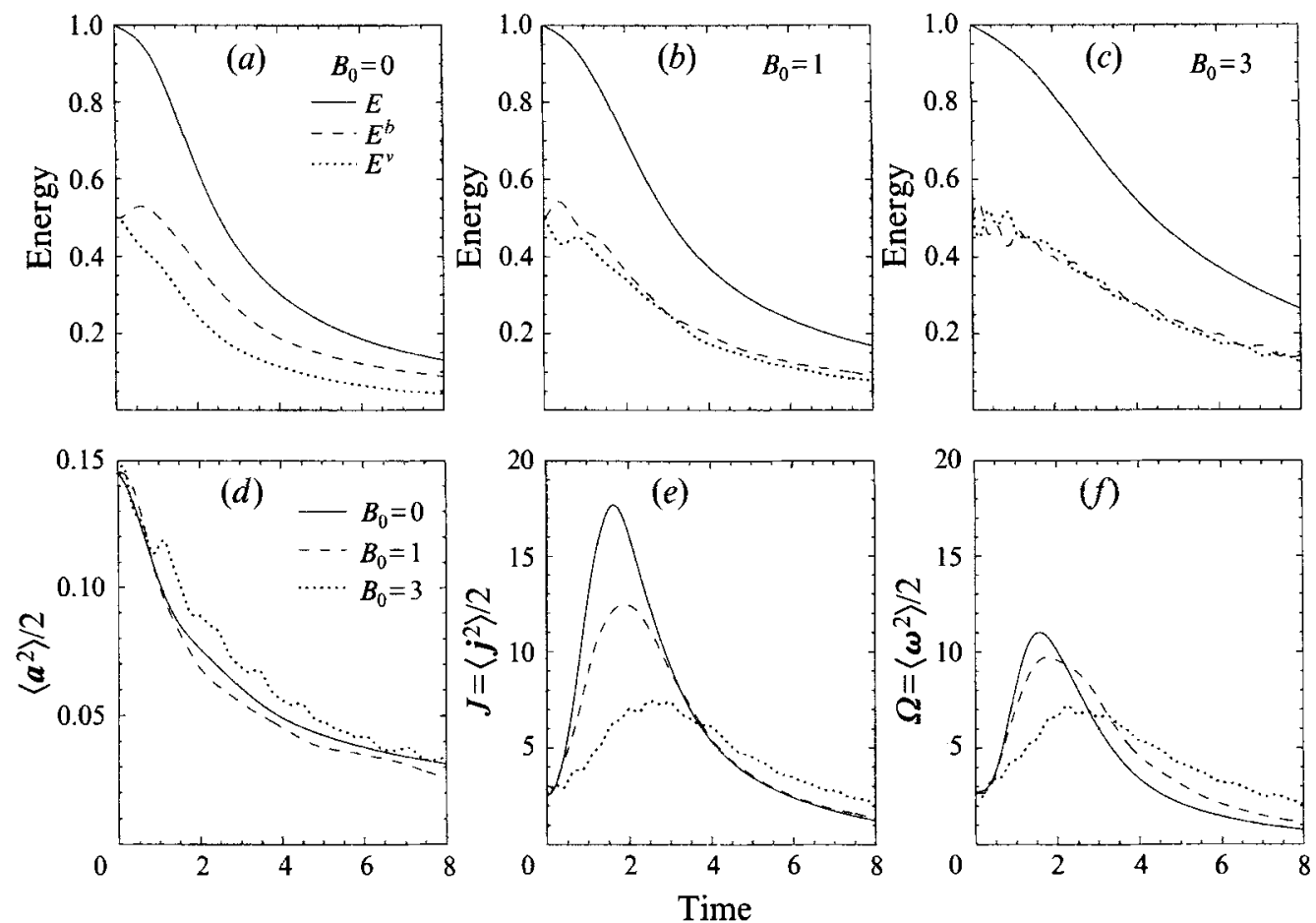

FIGURE 3. Time histories of some bulk quantities from runs c9, c11, and cl2 ( $\nu, \eta=1 / 200):(a-c)$ energies for each run, $(d)$ mean-square vector potential, $(e)$ mean-square current density, $(f)$ enstrophy.

we would wish. Nonetheless, the clear maxima in $\Omega$ and $J$ suggest that a (brief) period of turbulent dynamics does indeed occur, particularly for the lower- $B_{0}$ runs. Note that $\Omega$ and $J$ are plotted using the same vertical scaling to better emphasize their differences when $B_{0}=0$ and 1 , and their similarities when $B_{0}=3$.

Third and finally, the time histories display more structure (or 'fast' fluctuations) as $B_{0}$ is increased, this being particularly noticeable for $\left\langle a^{2}\right\rangle / 2$ which is the lowest $k$-moment sum shown. This suggests that there may be an increased oscillatory contribution in such quantities. Since the frequency of Alfvén waves is proportional to $B_{0}$, this is at least plausible. However, frequency-domain power spectra for $\left\langle a^{2}\right\rangle / 2, J$, and $\Omega$ show no strong peaks away from the zero frequency one. This may be because the oscillations are masked by the overall decay of these globals. 'Detrending' of the data before the frequency Fourier analysis is performed may be called for (e.g. Blackman \& Tukey 1958).

Having considered the flows in terms of time histories of the globals, we now investigate the anisotropies which develop in the presence of $B_{0}$. In order to quantify the degree of anisotropy associated with a flow, we introduce the generalized Shebalin (see SMM) angles, $\theta_{Q}$, defined by the relation

$$
\tan ^{2} \theta_{Q}=\frac{\sum k_{\perp}^{2}|Q(k, t)|^{2}}{\sum k_{z}^{2}|Q(k, t)|^{2}}
$$

where $k_{\perp}^{2}=k_{x}^{2}+k_{y}^{2}, Q$ is any one of the vector fields $\psi, v, \omega, \boldsymbol{a}, \boldsymbol{b}, j$, and the summations extend over all values of $k$. Physically, $\tan ^{2} \theta_{Q}$ may be interpreted as the ratio of a weighted mean-square perpendicular wavenumber to its parallel counterpart, the weighting factor being the "energy' spectrum for $Q$. With this definition, an isotropic 

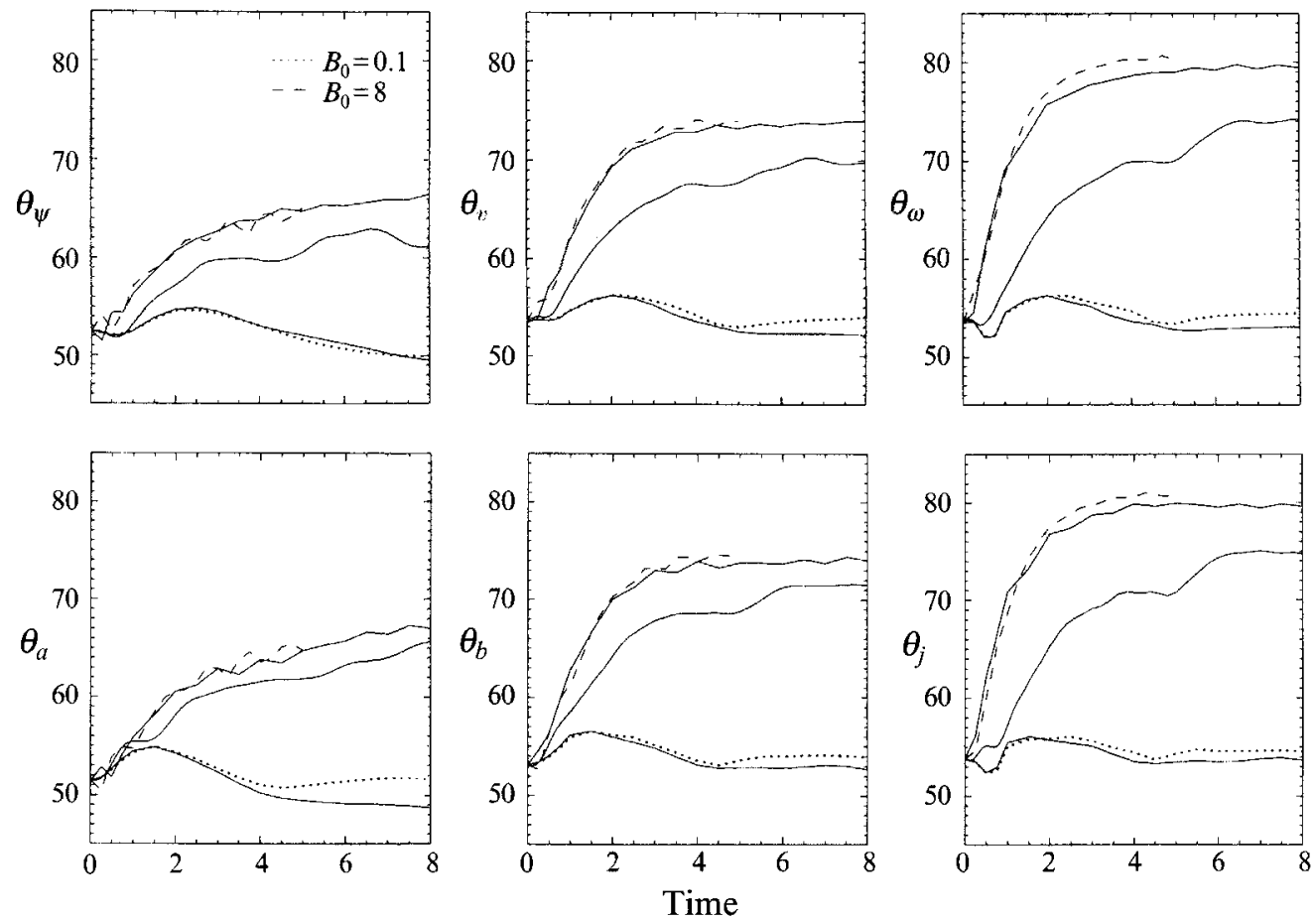

FIGURE 4. Generalized Shebalin angles for the C2 runs. Each curve (solid or broken) represents a distinct value of $B_{0}$. In general the curves are ordered by their value of $B_{0}$, larger values corresponding to stronger anisotropies. However, where ambiguity is possible (i.e. $B_{0}=0,0.1$ and $B_{0}=3,8$ ) unique line styles are used as shown in the legend. In this and later figures all angles are in degrees.

spectrum corresponds to $\theta_{Q}=\tan ^{-1} \sqrt{ } 2 \approx 54.74^{\circ}$, whereas a spectrum having all its energy in modes perpendicular to $B_{0}$ has $\theta_{Q}=90^{\circ}$. As a shorthand we will speak of $Q$ increasing or decreasing, where we define the orderings as $\psi<v<\omega$ and $a<b<j$.

In figure 4 plots of $\theta_{Q}$ for each field are shown as a function of time and $B_{0}$ for the $\mathrm{C} 2$ runs. Although the angles fluctuate, their initial behaviour when $B_{0} \gtrsim \frac{1}{2}$ is generally characterized by a more or less steady increase with time. At later times, and when $B_{0} \gtrsim 1$, most of the angles attain approximately constant values; but note that the time taken to reach the plateaus appears to depend on both $Q$ and $B_{0}$. It is also evident that the degree of anisotropy increases with $B_{0}$, at least for $B_{0} \lesssim 3$. Increasing $B_{0}$ above this value does not appear to markedly alter the degree of anisotropy which develops in the flows. This saturation can also be seen in figure 5, which displays the $v$-related angles at a fixed time $(t=2)$ as a function of $B_{0}$. The $b$-type angles behave in a very similar fashion. We defer discussion on possible reasons for the saturations with time and $B_{0}$ to $\S 8$. Figure 4 also suggests that there is a minimum value of $B_{0}$ below which the anisotropy does not develop. The qualitative features pointed out in this paragraph were also seen in SMM's earlier study of two-dimensional MHD turbulence.

Considering the runs individually for the moment, there are two points we wish to remark upon. First, for runs with $B_{0} \gtrsim \frac{1}{2}$ it is usually the case that $\theta_{\psi}<\theta_{v}<\theta_{\omega}$ and that $\theta_{a}<\theta_{b}<\theta_{j}$, orderings also observed by SMM who attributed them to increased anisotropy at higher wavenumbers. To help understand this, consider the $v$-related angles and fields: The modal relations

$$
\begin{aligned}
|\boldsymbol{v}(\boldsymbol{k})|^{2} & =k^{2}|\boldsymbol{\psi}(\boldsymbol{k})|^{2}, \\
|\omega(\boldsymbol{k})|^{2} & =k^{2}|\boldsymbol{v}(\boldsymbol{k})|^{2},
\end{aligned}
$$




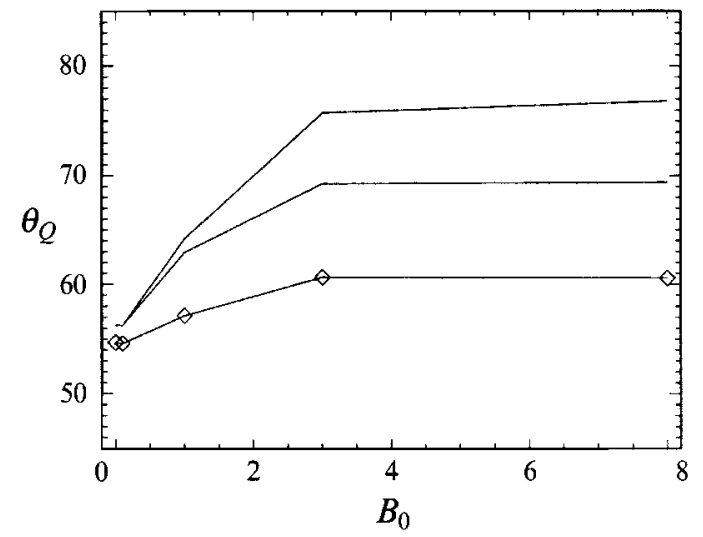

FIgURE 5. The $v$-related Shebalin angles as a function of $B_{0}$ at $t=2$ for the $C 2$ runs. Note that $B_{0} \equiv B_{0} /\left\langle b^{2}(t=0)\right\rangle^{1 / 2}$. From bottom to top the curves are for $Q=\psi, v$, and $\omega$, respectively. The diamonds indicate the actual numerical data points.
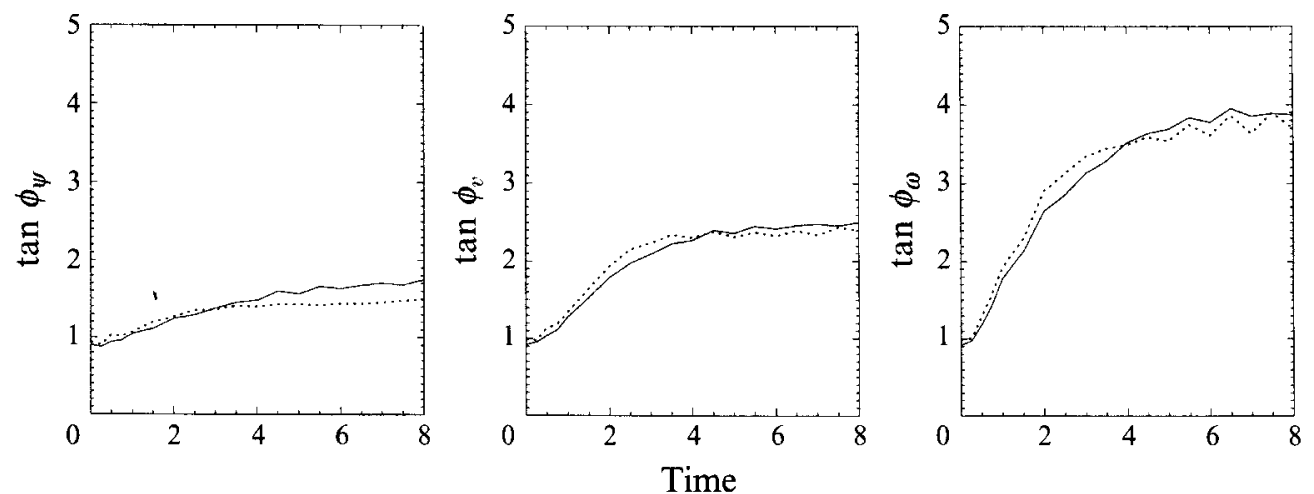

FIGURE 6. Ratios of r.m.s. wavenumbers for run $\operatorname{c12}\left(B_{0}=3\right)$. The solid curves are for $\alpha=x$ and the dotted ones for $\alpha=y$. Again only the $v$-type angles are displayed.

show that the velocity is more strongly dependent on the small-scale structure of the turbulence than is the stream function, while the vorticity's dependence is stronger still. Thus, if the anisotropy is more pronounced at smaller scales we would expect to see the above ordering of angles. Exactly analogous arguments hold for the $b$-type angles. Using the shorthand mentioned above we may phrase this result as: for a given $B_{0}$, anisotropy increases as $Q$ does.

The stronger dependence on small-scale structure for $\theta_{\omega}$ and $\theta_{j}$ may also explain why, for strong $B_{0}$, these angles reach their plateau levels faster than the lower- $Q$ ones, despite having to achieve larger values: higher-wavenumber modes have shorter characteristic times and can thus achieve 'equilibrium' values faster than the lower- $k$ modes. For the $B_{0}=3$ and 8 runs, the $v-b$ and $\omega-j$ angles level out at $t \approx 4$, whereas the $\psi-a$ angles have apparently not done so by $t=8$. Furthermore, comparing only the rapid-rise portions of the curves, the $\omega-j$ angles reach $70^{\circ}$ in half the time it takes for the $v-b$ angles to do so. However, it should be noted that such behaviour might be a consequence of the low Reynolds numbers characterizing these flows.

The second point regarding the individual runs is not obvious from figure 4 , but plots of $\theta_{v}$ and $\theta_{b}$ on the same axes show that there is a small but definite tendency for $\theta_{b}$ to exceed $\theta_{v}$ when $\frac{1}{2} \lesssim B_{0} \lesssim 3$. We believe this to be a consequence of $r_{A}$ being 


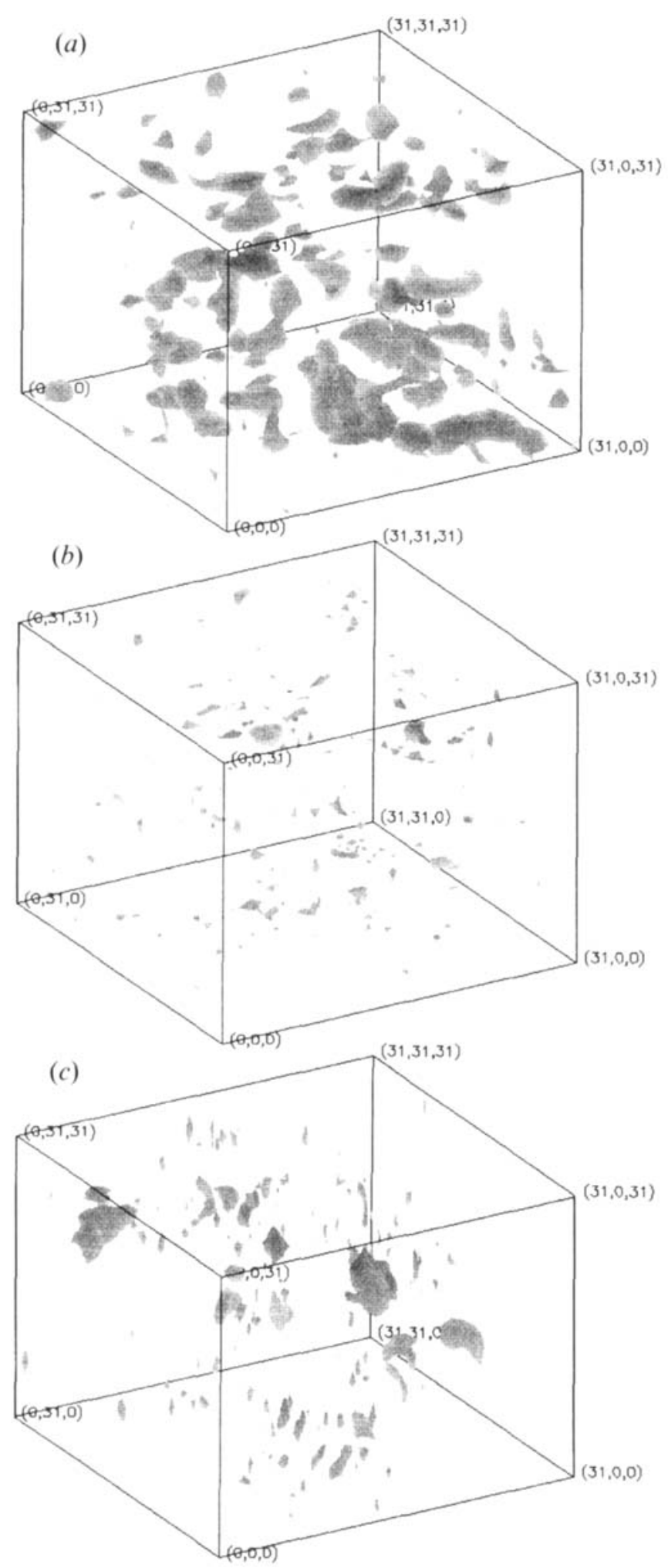

FIGURE 7. Isosurfaces for the squared electric current density. (a) The B1 initial conditions: the isotropy of the data is reflected in the absence of a preferred orientation direction for the tubes. 
somewhat less than unity, the slightly greater magnetic energy leading to bigger values of $\theta_{b}$ (this explanation also requires that $r_{A}(k)$ be a function of $k$, since simple proportionality of the kinetic and magnetic energy spectra would lead to equal Shebalin angles for the two fields). As noted above, $B_{0} \gtrsim 3$ appears sufficient to enforce near-exact equipartition of the kinetic and magnetic energies, which explains the disappearance of the effect at high $B_{0}$. Similar statements hold for $\theta_{\omega}$ and $\theta_{j}$, the ratio $\Omega / J$ being analogous to $r_{A}$.

While we have shown that significant anisotropies develop perpendicular to $B_{0}$, it is unclear as to whether the perpendicular behaviour is itself isotropic or perhaps dominated by contributions from, say, the $y$-components of the variables. The equations of motion do not obviously suggest anything other than isotropic behaviour in the perpendicular planes, but it will be as well to check this. In order to do so we utilize the original Shebalin angles (SMM), but with a change of notation,

$$
\tan ^{2} \phi_{Q \alpha}=\frac{\sum k_{\alpha}^{2}|Q(k, t)|^{2}}{\sum k_{z}^{2}|Q(k, t)|^{2}},
$$

where $\alpha=x$ or $y$, and, as above, $Q$ is any one of the six major fields. Clearly, fully isotropic flows are characterized by $\phi_{Q \alpha}=45^{\circ}$ for both values of $\alpha$. Furthermore, equal values of $\tan \phi_{Q x}$ and $\tan \phi_{Q y}$ indicate equal mean wavenumbers in the $x$ - and $y$-directions, and are thus consistent with isotropy in the perpendicular planes. As expected, time histories of these angles (not shown) indicate approximate equipartition of energy in the perpendicular plane, for all values of $B_{0}$. Thus, we judge that the turbulence remains isotropic in the perpendicular planes, despite the imbalance with respect to the parallel direction, and conclude that such two-dimensional isotropy is a natural feature of these runs.

We may also consider the spectral anisotropy from a slightly different perspective. Because the Shebalin angles are defined in terms of inverse tangents, the extent of the two-dimensionality relative to $\boldsymbol{B}_{0}$ is not always clear. We therefore include plots of $\tan \phi_{Q}$ versus time from run $\mathrm{cl} 2$; these represent the ratio of a mean perpendicular wavenumber to the parallel one, or equivalently the ratio of the parallel correlation length to a perpendicular one (figure 6). The ratios are modestly, but significantly, greater than unity. Indeed, the plot indicates that the ' $b$ '-field correlation length in the parallel direction is $\approx 2.5$ times that in a transverse direction. While this is well short of the ratios observed in experimental fusion devices ( $z 10$, see references in SMM), the trend is clearly the same (presumably the Reynolds numbers are higher in these fusion devices). Similarly, the solar wind observations indicate that the minimum variance direction for MHD-scale magnetic fluctuations is usually aligned with the mean magnetic field (Klein, Roberts \& Goldstein 1991).

Finally in this section, we consider the $x$-space manifestations of the anisotropy by displaying a few isosurface plots of the electric current density (figure 7). The difference between the $t=2$ isosurfaces for the $B_{0}=0$ and 3 runs is quite clear: in the former the surfaces are qualitatively similar to the initial conditions, being essentially unaligned blobs, whereas in the latter, there is a strong tendency for the tubes to be $(a)$ elongated, and $(b)$ aligned with the $\boldsymbol{B}_{\mathbf{0}}$ direction, consistent with the greater correlation length in this direction. Vorticity isosurfaces as a function of time and $B_{0}$ show similar behaviour.

(b) Run $9\left(B_{0}=0\right)$ at $t=2$; at this time the enstrophy and mean-square current are close to their maximum values. (c) Run $13\left(B_{0}=3\right)$ at $t=2$; the anisotropy has almost saturated by this time. Note the alignment of the tubes in the $z$-direction. The isosurface threshold in $(a-c)$ figures is set at $60 \%$, and the $\boldsymbol{z}$-direction is vertical. 


\section{Anisotropy: influence of $\nu, \eta$, and spatial resolution}

Having found that $B_{0} \neq 0$ can induce spectral anisotropy, it is of interest to investigate how this dynamical anisotropy varies as a function of the Reynolds numbers. Indeed, the main purpose of this section is to show that, for a given $B_{0}$, the flows become more anisotropic as the Reynolds numbers are increased. There is, however, a caveat. Because the larger simulations are computationally demanding most runs are, to varying extents, under-resolved. Table 1 indicates the maximum resolved wavenumber and the maximum value of $k_{d}(t)$ for each run. To assess the accuracy of under-resolved runs, we may compare runs c11 and 17, which initially differ only in their resolutions, the former being significantly better resolved than the latter.

Figure 8 displays plots of a few bulk quantities for these runs. In general, the agreement between the runs is qualitatively good, and indeed often quantitatively so, there being almost no visible difference in the energies for example. However, note the reduced and shifted maxima of $\Omega$ and $J$. Furthermore, when we consider the Shebalin angles (figure 9) clear discrepancies are also evident. Most important of these is the under-estimation of $\theta_{\omega}$ and $\theta_{j}$, that is, the angles most dependent on the small-scale structure of the turbulence (this consequence of under-resolution may explain why Hossain, Vahala \& Montgomery 1985 did not see the $\theta_{\psi}<\theta_{v}<\theta_{\omega}$ ordering in their study of forced two-dimensional MHD turbulence with a $B_{0}$ ). Since we are about to compare runs with different Reynolds numbers, we must keep in mind that quantities depending strongly on the high- $k$ components of the fields will be less accurately represented in the more under-resolved runs.

Consider runs $\mathrm{cll} 1 \mathrm{and} 16$, whose runtime parameters differ only in that the latter has half the resolution and double the dissipation coefficients of the former. As these two runs are almost resolved and also have $B_{0}=1$, they afford a clean opportunity to examine the Reynolds number dependence of the anisotropy. Figure 10 shows that the Shebalin angles characterizing these runs are always ordered such that $\theta_{Q}(\mathrm{c} 11)>\theta_{Q}(16)$ for given $B_{0}$, except occasionally at early times. Also plotted are the Shebalin angles for another $B_{0}=1$ run, namely c14; this has initial large-scale Reynolds numbers of 1000 , but is significantly under-resolved. With such a high Reynolds number flow we expect $\theta_{Q}(\mathrm{c} 14)>\theta_{Q}(\mathrm{c} 11)$; however, the figure shows that this is only true for $Q=\psi$ and $a$. It is our belief that this is entirely a consequence of the under-resolved nature of run c14, and this stands as a warning regarding the dangers associated with interpreting such runs. Runs 12 and 15 have similarities to runs $\mathrm{c} 11$ and $\mathrm{c} 14$, in the sense that the latter member of each pair is more under-resolved and has higher Reynolds numbers. Similar orderings of the Shebalin angles for these pairs are observed.

We may also compare corresponding runs from the B1 $(\nu, \eta=1 / 100)$ and $\mathrm{C} 2$ $(v, \eta=1 / 200)$ sets, which have quite different initial conditions in terms of spectral profiles and the initial values of $\Omega$ and $J$ for example (table 2). Notwithstanding these differences, the Shebalin angles are still ordered in terms of increasing Reynolds numbers, that is $\theta_{Q}(\mathrm{C} 2)>\theta_{Q}(\mathrm{~B} 1)$ for the same $B_{0}$. We therefore suggest that, provided the initial states are turbulent and approximately isotropic, flows with higher values of the Reynolds numbers will develop stronger anisotropies relative to $B_{0}$. While in these simulations there is no hint of any saturation of the anisotropies occurring beyond critical values of the Reynolds numbers, such a possibility cannot be ruled out. Furthermore, the role of the finite-volume periodic domain may be important. In particular, these boundary conditions do not permit propagating fluctuations to 

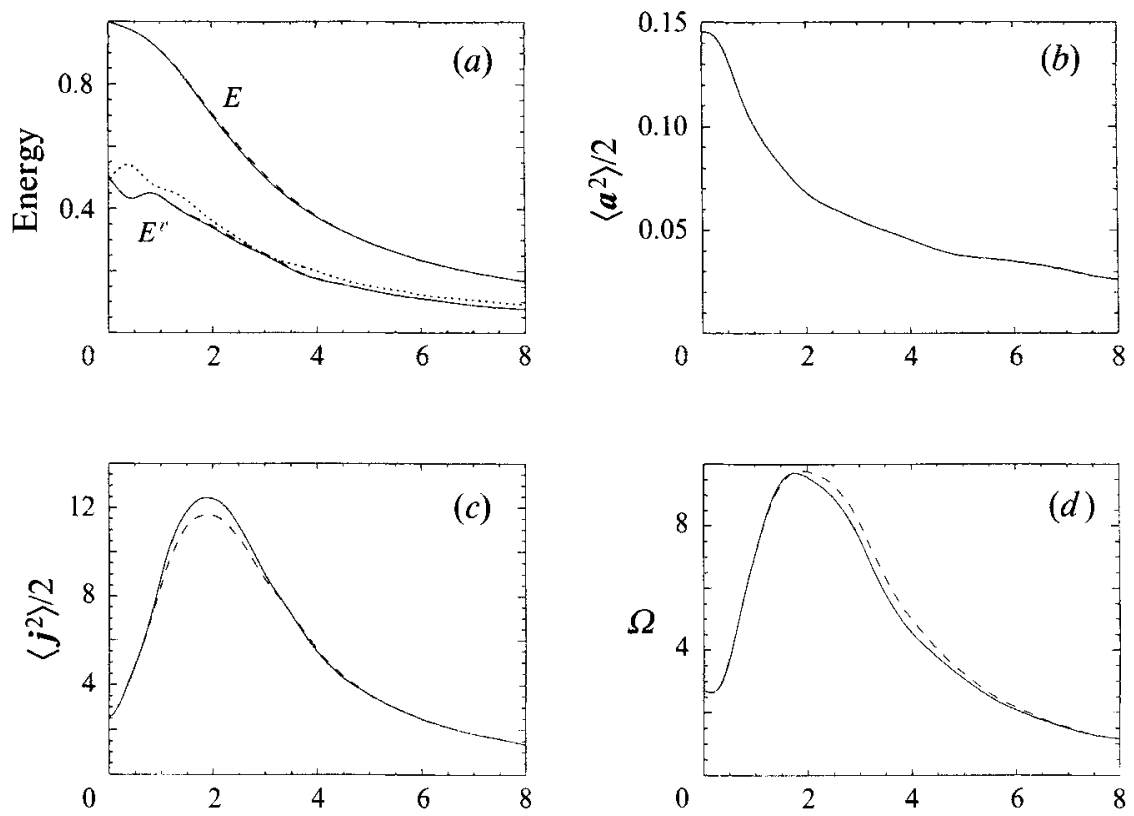

Time

FIGURE 8. Over-plots of selected global quantities for the almost resolved run c11 (solid curves) and the under-resolved run 17 (dashed). The dotted curve in $(a)$ is $E^{b}$ for run c11. At times the dashed curves overlie the solid ones.
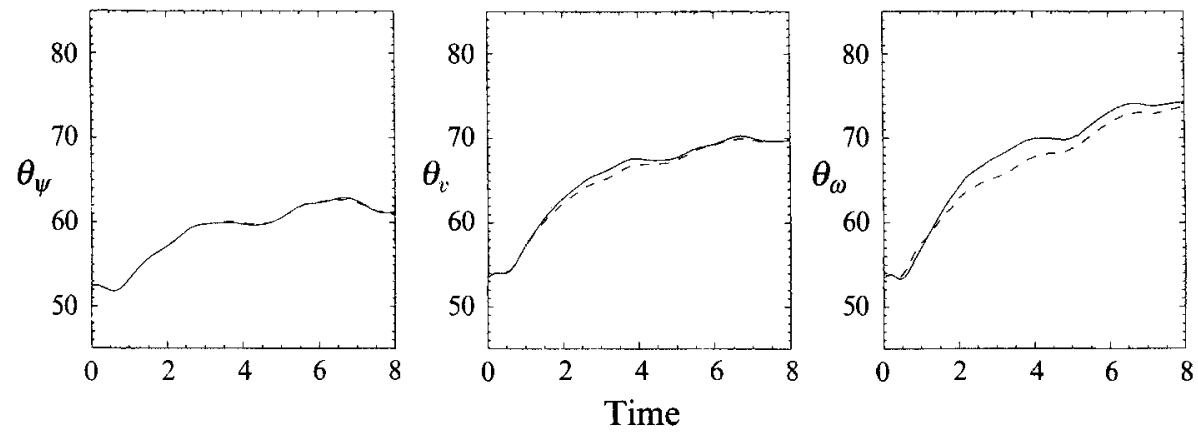

FIGURE 9. Comparison of some Shebalin angles for an almost-resolved run (c11, solid curves) and a clearly unresolved one (17, dashed curves). Aside from their resolution, these runs have identical initial parameters. Similar plots for the ' $b$ '-type angles are identical in character.

'escape' from the system, as they would in more realistic geometries. Nonetheless, at present we still agree with SMM, who stated that 'the smaller the dissipation coefficients, the greater the degree of anisotropy is likely to be'.

This section would not be complete without a few words regarding the differences between dissipative and non-dissipative runs. While we do not show the plots, comparison of the Shebalin angles for runs 3 and 8 is revealing (see figure 10 in SMM). For the first 1-2 characteristic times, $\theta_{Q}(3)$ and $\theta_{Q}(8)$ are graphically indistinguishable. Beyond this however, the angles part company. The run 3 angles behave in essentially the same way as the angles discussed in the previous section, initially continuing to rise and then plateauing at convincingly non-isotropic levels. In contrast to this, the nondissipative angles, $\theta_{Q}(8)$, decrease until attaining roughly the isotropic value and 

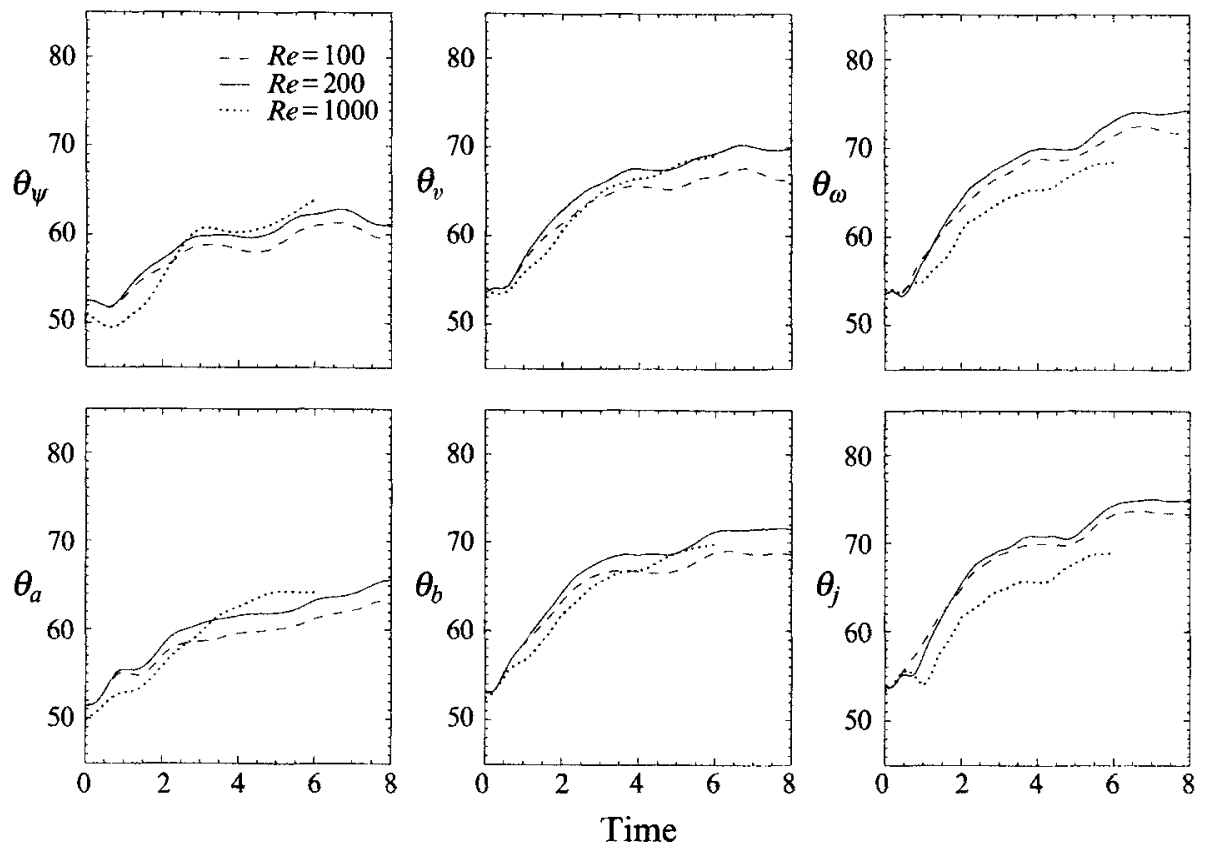

FIGURE 10. Comparison of some of the Shebalin angles for runs c11 (solid curves) and 16 (dashed). The runs differ in their values of $v$ and $\eta, 1 / 200$ for run $c 11$ and $1 / 100$ for run 16. The dotted curves are for run c14, which has $R e=R m=1000$, but is under-resolved.

thereafter fluctuate unsystematically about this level. Clearly, as discussed in SMM, sinks for the kinetic and magnetic energies appear to be essential if the anisotropy is to be maintained. If the resonance process outlined by SMM is responsible for the anisotropy, it would seem that higher-order effects swamp the weak-turbulence ones when $v=\eta=0$.

\section{Anisotropy: influence of rugged invariants}

Obviously the factors considered above are not the only ones which can influence development of anisotropy. In this section we chiefly discuss the impact of non-zero cross-helicity. A few comments regarding the influence of some other well-known quadratic flow parameters are also made. We should however make it clear that this section is neither complete in its coverage nor exhaustive of possibilities.

Cross-helicity. It is well known that increasing the normalized cross-helicity reduces the importance of the nonlinear terms in the equations of motion. More specifically, in high- $\sigma_{c}$ flows spectral transfer is directly weakened owing to the reduced strength of the nonlinear couplings. In fact, rigorously extremal $\sigma_{c}$ corresponds to $v= \pm b$, in which case the nonlinear terms exactly cancel, and there is no turbulence even when the Reynolds numbers are very large. Thus, for two flows $A$ and $B$ which have the same initial spectra but different phase relations such that $0<\sigma_{c}(A)<\sigma_{c}(B)<1$, we might expect both to be turbulent, but flow $B$ to be somewhat less so. As far as the development of anisotropy is concerned, non-zero $\sigma_{c}$ will presumably lead to somewhat less-anisotropic states, all other things being equal. While we do not show the appropriate plots, comparison of the Shebalin angles for the Al runs with those for the corresponding runs in the set $\mathrm{B} 1$ shows that this is indeed the case (when $B_{0} \gtrsim \frac{1}{2}$ ). Qualitatively speaking, the Shebalin angles for the two sets of runs are very similar. 


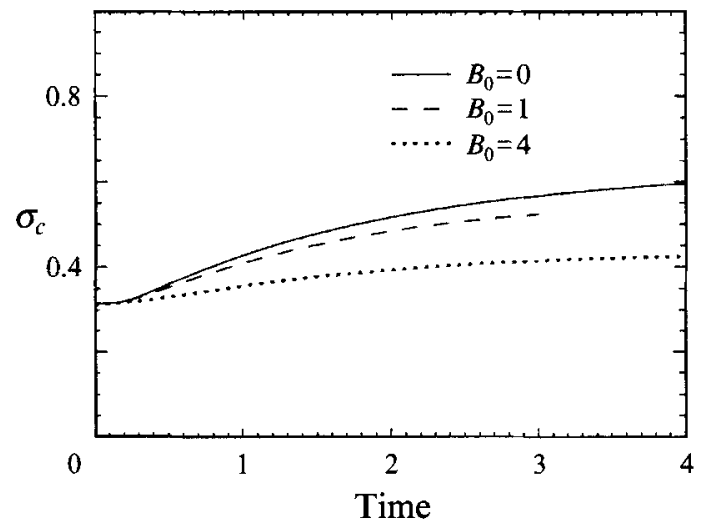

FIGURE 11. Normalized cross-helicity as a function of time for runs 1, 3 and 5 . Note the reduction in growth of $\sigma_{c}$ as $B_{0}$ is increased.

Quantitatively, however, the zero-cross-helicity runs tend to have larger values for a given $B_{0}$, with the differences becoming more pronounced as $Q$ increases.

Note that dynamic alignment (Dobrowolny, Mangeney \& Veltri 1980; Pouquet, Meneguzzi \& Frisch 1986) occurs in the A1 runs. This turbulent relaxation process is characterized by $\left|\sigma_{c}\right| \rightarrow 1$ as $t$ increases, even though both the energy and the crosshelicity are decaying. In other words, the cross-helicity decays more slowly than the energy, causing the flow to evolve towards a state where the nonlinear terms cancel. Figure 11 shows several examples from the A1 runs. Clearly, at least the characteristic timescale over which dynamic alignment takes place is affected by the value of $B_{0}$, and it may be that the final state is too. Various groups (Grappin et al. 1982; Pouquet et al. 1986; Ting, Matthaeus \& Montgomery 1986; Biskamp \& Welter 1989; Stribling $\&$ Matthaeus 1991) have noted that when $\left|\sigma_{c}\right| \lesssim 0.2$ dynamic alignment appears to operate only weakly, the growth of $\sigma_{c}$ being approximately logarithmic in time (Grappin et al. 1982). Our results support this viewpoint. For example, the B1 runs, which have $\sigma_{c}(t=0) \approx 0.01$, exhibit only very weak growth of $\sigma_{c}$ for $B_{0}<1$, and $\sigma_{c}$ actually reverses sign for larger values of $B_{0}$.

Magnetic helicity. As mentioned in the introduction, Stribling, Matthaeus, and coworkers (Stribling et al. 1994a,b) recently investigated the behaviour of threedimensional MHD flows with non-zero values of $H_{m}$. Briefly, they found that the turbulent magnetic helicity nonlinearly decays toward zero, while a similar quantity $\left(\propto B_{0} \cdot \int_{0}^{t} E_{0}(x, t) \mathrm{d} t\right.$ where $E_{0}=-\langle\boldsymbol{v} \times \boldsymbol{b}\rangle$, is the volume averaged induced electric field) grew at $H_{m}$ 's expense. The process continued until essentially no turbulent magnetic helicity remained, typically taking 5-10 Alfven times to complete. At this point the flow is in a state similar to the initial states investigated here, provided that the Reynolds numbers are still large enough to maintain a turbulent flow. We therefore conjecture that similar anisotropies with respect to $B_{0}$ will develop when $H_{m} \neq 0$, but perhaps in a different fashion to that described herein while the redistribution of magnetic helicity is occurring (e.g. faster, slower, delayed onset). We stress that the nonlinear decay of $H_{m}$ is a consequence of its conversion into another magnetichelicity-type quantity, and is not due to the dissipation of $H_{m}$ (Stribling et al. 1994a,b).

Alfvén ratio. While not actually a rugged invariant, the initial value of this parameter may have some effect on the development of the anisotropy. In particular, we expect values less than unity to be associated with greater anisotropy of the $b$-related fields relative to the corresponding $v$ ones, e.g. $\theta_{j}>\theta_{\omega}$. As discussed above, initial states with 
$r_{A}=1$ exhibited this behaviour when $B_{0}$ took values strong enough to induce the anisotropy, but not so strong that it also imposed equipartition of kinetic and magnetic energy. However, even if $r_{A}$ is initially far from unity, the situation is unlikely to persist for long since when $B_{0} \neq 0$ the Alfvén effect will soon enforce $r_{A} \approx 1$ (but see Biskamp \& Welter 1989 and Stribling \& Matthaeus 1991). Thus, we should not expect to see major differences in $\theta_{\imath}$ and $\theta_{j}$ (for example) enduring beyond an Alfvén time or two. In other words, the correlation lengths for $\Omega$ and $J$ should become fairly similar if they are not so initially, and similarly for $E^{v}$ and $E^{b}$.

Kinetic helicity. Define this by $H_{k}=\langle\boldsymbol{v} \cdot \omega\rangle$. This too is not a rugged invariant, but since the momentum equation may be written in a form containing the term $v \times \omega$, the kinetic helicity is another quantity whose presence tends to reduce the strength of the nonlinear couplings. In other words, $H_{k}$ non-zero tends to make the flow less turbulent than it would be otherwise (ignoring possible dynamo effects). Thus, we suggest that just as $\sigma_{c} \neq 0$ reduces the degree of anisotropy associated with a particular $B_{0}$, so too will $H_{k} \neq 0$. Naturally this will need to be verified.

\section{Other consequences of $\boldsymbol{B}_{0}$}

There are several other ways in which $\boldsymbol{B}_{0}$ influences the development of a turbulent flow, aside from its anisotropy-inducing effects. We now consider some of these.

Maxima and suppression of turbulence. The maxima of the enstrophy, $\Omega$, and the mean-square current density, $J$, decrease essentially monotonically as $B_{0}$ increases (cf. figure 3). Since growth of $\Omega$ and $J$ is an indicator of the degree of turbulence developed in the flow, the decreasing maxima suggest that a d.c. magnetic field suppresses turbulence ever more strongly as its strength increases. In fact, naive examination of the equations of motion indicates that when $\boldsymbol{B}_{\mathbf{0}}$ is large the linear terms dominate the nonlinear ones, leading to the well-known result that $v$ and $\boldsymbol{b}$ obey (dissipative) wave equations. However, this is not the whole story since the approximation fails to correctly account for the different perpendicular and parallel correlation lengths induced by $B_{0}$.

As noted by Frisch et al. (1983), imposing a sufficiently strong $\boldsymbol{B}_{0}$ suppresses development of small-scale structures in that direction. It was their conjecture that this is a consequence of the lack of $X$-type neutral points in such flows, such sites being associated with intense generation of small-scale structures (e.g. $\omega$ and $j$ ). Whatever the reason, it does not follow that complete suppression of the turbulence ensues for large enough $\boldsymbol{B}_{0}$. By analogy with neutral fluid flows, where strong rotation can induce two-dimensionality of the flow (with respect to the rotation axis), it is physically plausible that an applied $\boldsymbol{B}_{0}$ might do likewise for magnetofluids. In other words, the initially three-dimensional MHD turbulence could be reduced to largely decoupled planes of two-dimensional MHD turbulence, oriented perpendicular to $\boldsymbol{B}_{0}$ (e.g. Cowling 1957). The isotropy inferred to be present in the perpendicular planes of the flows discussed herein is consistent with such a possibility, as are the disparate parallel and perpendicular correlation lengths. We should also note that recent work on nearly incompressible MHD (Zank \& Matthaeus 1992a,b, 1993) shows that in the limit of strong $\boldsymbol{B}_{0}$, the compressible three-dimensional MHD equations reduce to incompressible two-dimensional equations similar to those just discussed. So it would seem that all roads do lead to Rome in the sense that $(a)$ physically based arguments, $(b)$ mathematical theorems and results, and $(c)$ numerical simulations all indicate or suggest the same behaviour.

Recall that when such incompressible two-dimensionality does ensue, $\boldsymbol{B}_{0}$ no longer 
plays a dynamical role for flow in the perpendicular planes, at least to leading order. This fact has been used to justify investigation of purely two-dimensional MHD turbulence with no $\boldsymbol{B}_{\mathbf{0}}$ (Biskamp \& Welter 1989). While our results are not conclusive, we believe they provide further numerical support for this approach. Once simulations of sufficient size to resolve possible inertial ranges in the parallel and perpendicular directions are practical we will be in a much better position to test this belief.

Alfven ratio. We have already discussed this quantity in several places above, so that here we make only a few additional points. With no d.c. field present, the initial equipartition of kinetic and magnetic energy disappears in a fraction of an eddy turnover time. In fact, the Alfvén ratio quickly evolves towards values which are consistently and significantly less than unity, indicating development of an excess of magnetic energy over kinetic. Typically, $r_{A} \approx 0.7$ for $B_{0} \leqslant \frac{1}{2}$, with $r_{A}(k)$ being very roughly flat away from $k_{\min }$ and $k_{\max }$.

Such an 'excess' of magnetic energy has been seen in many other simulations (Pouquet et al. 1976; Pouquet \& Patterson 1978; SMM; Matthaeus \& Lamkin 1986), and is also regularly observed in solar wind data (Matthaeus \& Goldstein 1982; Roberts et al. 1987a,b). While no completely satisfactory explanation for this behaviour has yet been put forward, there are several candidates. Pouquet et al. (1976) argued that $r_{A} \lesssim 1$ is a consequence of local (in $k$-space) dynamo-type action, whereas Matthaeus \& Lamkin (1986) suggested that it may be a consequence of the dynamics associated with small-scale magnetic reconnection (in particular, the formation of electric current filaments and vorticity quadrupole structures near the $X$-type neutral points). Because the simulations reported here have low Reynolds numbers, we have not attempted to verify the correctness of either of these theories.

Biskamp \& Welter (1989) have argued that since no kinetic invariants exist for MHD (two- or three-dimensional), the Alfvén ratio should decrease as a function of time (assuming $v=\eta$ ), $E^{v}$ decaying faster than $E^{b}$ because some magnetic energy is inversecascaded with the magnetic helicity and thus preserved against dissipation, which is most effective at small scales. This is essentially a selective decay argument (Matthaeus \& Montgomery 1980; Ting et al. 1986); however, for two reasons it does not apply here. First, our runs have very little magnetic helicity and therefore the inverse cascade is only weakly active. Second, the presence of $B_{0}$ destroys the rugged invariance of $H_{m}$, thus removing the basis for its inverse cascade; indeed, as noted above, recent work (Stribling et al. 1994a, b) indicates that what turbulent magnetic helicity there is is nonlinearly converted into another form which may or may not be associated with reduced dissipation of $E^{b}$.

In concert with the development of 'excess' $E^{b}$, it is also the case that $\Omega / J \lesssim 1$, despite the initial (near) equivalence of $\omega$ and $j$, both spectrally and in bulk terms. In particular, for runs with $B_{0} \leqslant 1$, the maximum value of $J$ can exceed that of $\Omega$ by over $25 \%$ (cf. figure 3 ). It is not necessary that 'excess' $E^{b}$ (in the sense that it leads to values of $r_{A} \leqslant 1$ ) will automatically yield 'excess' $J \sim\left\langle(\nabla \times b)^{2}\right\rangle$, relative to $\Omega$. However, it appears that this is indeed the case, at least for initial spectral distributions of $\omega$ and $j$ which differ essentially only in their phases. We have already noted above that at higher values of $B_{0}$ the Alfvén effect comes into play, leading to approximate equipartition of the turbulent kinetic and magnetic energies. Increasing $B_{0}$ also causes $\Omega / J$ to approach unity.

Kinetic and magnetic helicities. While both these quantities are initially small, and indeed remain so throughout the runs, their time histories do show dependencies on $\boldsymbol{B}_{0}$. In particular, frequency-domain power spectra for $H_{m}$ and $H_{k}$ show pronounced peaks at even multiples of $B_{0}$. As noted above, Alfvén waves have a frequency proportional 
to $B_{0}$ so that one possible interpretation of the power-spectra data is that Alfvén waves are important for these two quantities.

\section{Summary and discussion}

We have found that a d.c. magnetic field $\left(\boldsymbol{B}_{0}\right)$ imposed on three-dimensional MHD turbulence induces enhanced transfer of excitations to perpendicular modes, relative to parallel ones. This anisotropy, as measured by ratios of correlation lengths, tends to increase with (i) $B_{0}$ (saturation occurs for a value $\gtrsim 3$ ), (ii) wavenumber (power of $k$ in $Q$ ), (iii) $R e$ and $R m$, (iv) time (with a saturation depending on $Q$ and $R e, R m$ ), and (v) decreasing $\sigma_{c}$. Furthermore, increasing $B_{0}$ suppresses the turbulence in the sense that it reduces $(a)$ the relative strength of the nonlinear couplings, $(b)$ the maxima of $\Omega$ and $J$, and $(c)$ the effectiveness of dynamic alignment (which itself contributes to $(a)$ ).

The behaviour of factors (i)-(iv) is in almost complete qualitative agreement with the two-dimensional results of SMM. The slight discrepancy concerns the value of $B_{0}$ at which anisotropy saturation occurs. While this 'critical' value is not determined precisely in either investigation, the three-dimensional value is almost certainly greater than the two-dimensional one. Given that there is an additional perpendicular degree of freedom in the three-dimensional geometry, this is quite reasonable.

The reasons for the saturations with $B_{0}$ and time are unclear to us. The latter may be a consequence of the low Reynolds numbers used here. For example, if the flows were fully turbulent for say 20 characteristic times instead of the $\approx 3$ we have here, saturation with time might occur at both a later time and a higher level. In other words, the lack of sustained turbulence in the flows may be prematurely curtailing the degree of anisotropy which develops. We intend to investigate this possibility using randomly forced turbulence. The saturation with $B_{0}$ is perhaps more fundamental, and may be related to the relatively decreased strength of the nonlinear couplings at high $B_{0}$.

Before proceeding with the discussion we address a point relating to the nature of the numerical solutions. Because we are considering isotropy versus anisotropy in this paper, it might be thought that a spherical truncation in Fourier space should be performed (as was done by SMM). There are, however, several arguments as to why this does not seem to be necessary. First, a few such truncation runs have been performed, wherein modes with $k^{2} \geqslant(N / 2)^{2}$ are maintained at zero. The initial conditions for these runs were identical to some of those used in the non-truncated runs discussed above. In each case the Shebalin angles for corresponding runs differed by less than $0.8 \%$, being all but visually indistinguishable when plotted on the same axes. Similar agreement is seen for the bulk parameters.

Second, dissipative simulations with no spherical truncation and $B_{0} \approx 0$ remain isotropic at all times investigated, suggesting that modes outside the truncation sphere (but within the cube) do not cause significant departures from isotropy, at least for the runs reported on here. In fact, 'corner' modes where $\boldsymbol{k} \approx(N / 2, N / 2, N / 2)$ make an isotropic contribution to the Shebalin angles so that their inclusion leads to underestimation of the anisotropies, although in view of the first point above the effect is not significant here. These points suggest that isotropic truncation is not essential for maintaining high accuracy in simulations with modest Reynolds numbers, such as those considered here.

As noted in the introduction, the work described in this paper is complementary to that of the earlier (analytic) study of Moffatt (1967). Using our notation, his results apply for the time range $0<t \ll 1$, with $R e \gg 1, R m \ll 1, b(t=0)=0$, and $v(t=0)$ turbulent, whereas our simulations have both $v$ and $b$ initially turbulent, and at best 
$R e=R m=200$ with $0<t \lesssim 10$. It follows that no direct comparison of the two studies is possible. Unfortunately, even if it were, the Reynolds numbers achieved herein are still far too small to support resolution of the various power-law scalings of $E^{v}(t)$ and $E^{b}(t)$ which emerge from Moffatt's analysis.

While the two studies have considered the development of anisotropy from different perspectives, we do wish to remark on Moffatt's prediction regarding the variances of the velocity components. With $v=\left(v_{x}, v_{y}, v_{z}\right)$, his calculations indicated that $W=$ $3\left\langle v_{z}^{2}\right\rangle /\left\langle v^{2}\right\rangle$ should increase from unity at $t=0$, to $\frac{3}{2}$ before $t=1$, that is an 'excess' of $\left\langle v_{z}^{2}\right\rangle$ should develop. Moffatt referred to this as partial 'channelling' of the kinetic energy into the component parallel to $\boldsymbol{B}_{0}$. We too saw a tendency for such an excess to occur, not just at early times but throughout the simulations, although in our case $W$ usually took values closer to 1 than $\frac{3}{2}$. Similar behaviour was observed for the analogous magnetic quantity. It may therefore be the case that this type of anisotropy generated during the early-time linear phase of the dynamics - also persists well into the nonlinear phase.

There is a somewhat subtle point that arises regarding the relationship between incompressibility, which is assumed here, and the role of anisotropy, which emerges dynamically in the present study. Depending upon the desired application, we might view the magnetofluid as a conducting ideal gas that is behaving incompressibly, or more precisely, nearly incompressibly. Although this type of behaviour is frequently treated as a simple consequence of low Mach number, a more precise characterization of the approach of compressible dynamics to incompressible dynamics is a somewhat delicate matter. Zank \& Matthaeus (1993, see also references therein) have examined the asymptotic low-Mach-number approach of a compressible ideal gas magnetofluid to dynamical states of near incompressibility.

For high plasma $\beta$, solutions to the three-dimensional incompressible MHD equations, identical to those employed here, are recovered as the leading-order dynamical behaviour, subject to certain bounds on the compressive component of the initial data. For this case, the results we find here carry over directly. However, for plasma $\beta$ of order unity or smaller, Zank \& Matthaeus (1993) found that geometrical restrictions on the initial data are also required in order to recover leading-order incompressible solutions. In particular, initial data giving rise to this type of nearly incompressible dynamics are restricted to being highly anisotropic, with the excited wave vectors being those almost perpendicular to the applied magnetic field. This is, of course, the same kind of anisotropy we observe in the incompressible framework. Thus, it is not at all clear that the present results have immediate consequences for $\beta \lesssim 1$ compressible MHD. In particular, the process of obtaining the incompressible limit seems to require the anisotropy that we find only after assuming that incompressibility is already obtained. On the other hand, we cannot rule out the possibility that anisotropy of the type we describe here emerges in these cases even when we do not enforce strict adherence to the conditions that give rise to incompressibility. Thus, for low- $\beta$ MHD, which is of great importance in space physics and astrophysics, it remains a matter of considerable interest to further investigate the dynamical emergence of anisotropy for compressible MHD, both in the incompressible limit and in the highly compressive regime.

Having established that two- and three-dimensional incompressible systems are largely equivalent as far as the development of $\boldsymbol{B}_{\mathbf{0}}$-induced anisotropy is concerned, further extensions to work along these lines can perhaps be most effectively carried out using two-dimensional codes with $\boldsymbol{B}_{\mathbf{0}}$ in the plane. This would of course allow investigation of flows with much higher Reynolds numbers. However, a possible 
drawback to this approach is the inherent one-dimensionality of the perpendicular direction for such two-dimensional systems. Quite how important $\boldsymbol{B}_{0}$ 's suppression of turbulence is in high Reynolds number flows, both forced and unforced, remains to be determined. In this context, the implications for current sheet formation and structure, and also for reconnection, are of theoretical and physical interest.

Finally, we comment on SMM's point regarding the implications of self-generating anisotropy for MHD turbulence theory. They noted that closures (DIA, EDQNM, TFM, etc.) and cascade analyses almost always assume isotropy, with extension to anisotropic cases being quite involved or even intractable. If, however - as physical insight, mathematical analysis, and simulations suggest - there is indeed a reduction of three-dimensional MHD turbulence to decoupled planes of isotropic twodimensional turbulence (at leading order), the news is not all bad. Direct numerical simulations of strictly two-dimensional MHD turbulence at $R e, R m \sim 10^{5}$ are almost within practical reach.

We would like to acknowledge Troy Stribling's contribution to this work, for it was he who wrote the initial version of the code. We wish to thank $\mathrm{H}$. K. Moffatt for bringing his 1967 paper to our attention, and an anonymous referee for helpful criticism of the paper. This work was supported by the UK SERC at St Andrews, and the United States NSF SPTP grant at Bartol. Computations were performed using the Cray Y/MP at the San Diego Supercomputing Centre, the St Andrews Sun workstations, and the Bartol VAXcluster.

\section{REFERENCES}

Biskamp, D. \& WelteR, H. 1989 Dynamics of decaying two-dimensional magnetohydrodynamics turbulence. Phys. Fluids B 1, 1964.

Blackman, R. B. \& Tukey, J. W. 1958 The Measurement of Power Spectra. Dover.

Braginskil, S. I. 1965 Transport processes in a plasma. Rev. Plasma Phys. 1, 205.

Canuto, C., Hussaini, M. Y., Quarteroni, A. \& Zang, T. A. 1988 Spectral Methods in Fluid Mechanics. Springer Series in Computational Physics.

Cowling, T. G. 1957 Magnetohydrodynamics. Interscience.

Dobrowolny, M., Mangeney, A. \& Veltri, P. 1980 Fully developed anisotropic hydromagnetic turbulence in interplanetary space. Phys. Rev. Lett. 45, 144.

Domaradzki, J. A., Rogallo, R. S. \& Brachet, M. E. 1993 An analysis of subgrid-scale interactions in numerically simulated isotropic turbulence. Phys. Fluids A 5, 1747.

Frisch, U., Pouquet, A., Léorat, J. \& Mazure, A. 1975 Possibility of an inverse cascade of magnetic helicity in magnetohydrodynamic turbulence. J. Fluid Mech. 68, 769.

Frisch, U., Pouquet, A., Sulem, P.-L. \& Meneguzzi, M. 1983 The dynamics of two-dimensional ideal mhd. J. Méc. Théor. Appl. 216, 191.

Gottlieb, D. \& Orszag, S. A. 1977 Numerical Analysis of Spectral Methods: Theory and Applications. SIAM.

Grappin, R., Frisch, U., Léorat, J. \& Pouquet, A. 1982 Alfvénic fluctuations as asymptotic states of MHD turbulence. Astron. Astrophys. 105, 6.

HOLLWEG, J. V. 1985 Viscosity in a magnetized plasma: physical interpretation. J. Geophys. Res. 90, 7620.

HollweG, J. V. 1986 Viscosity and the Chew-Goldberger-Low equations in the solar corona. Astrophys. J. 306, 730.

Hossain, M., Vahala, G. \& Montgomery, D. 1985 Forced magnetodynamic turbulence in a uniform external magnetic field. Phys. Fluids 28, 3074.

Klein, L. W., Roberts, D. A. \& Goldstein, M. L. 1991 Anisotropy and minimum variance directions of solar wind fluctuations in the outer heliosphere. J. Geophys. Res. 96, 3779. 
Kraichnan, R. H. 1965 Inertial-range spectrum of hydromagnetic turbulence. Phys. Fluids 8, 1385.

KraichNaN, R. H. 1967 Inertial ranges in two-dimensional turbulence. Phys. Fluids 10, 1417.

Kraichnan, R. H. 1973 Helical turbulence and absolute equilibrium. J. Fluid Mech. 59, 745.

Kraichnan, R. H. \& Montgomery, D. C. 1980 Two-dimensional turbulence. Rep. Prog. Phys. 43, 547.

Matthaeus, W. H. \& Goldstein, M. L. 1982 Measurement of the rugged invariants of magnetohydrodynamic turbulence in the solar wind. J. Geophys. Res. 87, 6011.

Matthaeus, W. H. \& Lamkin, S. L. 1986 Turbulent magnetic reconnection. Phys. Fluids 29, 2513.

Matthaeus, W. H. \& Montgomery, D. 1980 Selective decay hypothesis at high mechanical and magnetic Reynolds numbers. Ann. NY Acad. Sci. 357, 203.

MoffatT, H. K. 1967 On the suppression of turbulence by a uniform magnetic field. J. Fluid Mech. 28, 571 .

Montgomery, D. C. 1982 Major disruption, inverse cascades, and the Strauss equations. Physica Scripta T1/2, 83.

ORsZAG, S. A. 1971 Numerical simulation of incompressible flows within simple boundaries: I. Galerkin (spectral) representations. Stud. Appl. Maths 50, 293.

PARKer, E. N. 1958 Dynamics of the interplanetary gas and magnetic fields. Astrophys. J. 123, 644.

PARKer, E. N. 1979 Cosmical Magnetic Fields: Their Origin and Activity. Oxford University Press.

Patterson, G. S. \& OrSzaG, S. A. 1971 Spectral calculations of isotropic turbulence: efficient removal of aliasing interactions. Phys. Fluids 14, 2538.

Pouquet, A., Frisch, U. \& LÉorat, J. 1976 Strong mhd helical turbulence and the nonlinear dynamo effect. J. Fluid Mech. 77, 321.

Pouquet, A., Meneguzzi, M. \& Frisch, U. 1986 Growth of correlations in magnetohydrodynamic turbulence. Phys. Rev. A 33, 4266.

Pouquet, A. \& Patterson, G. S. 1978 Numerical simulation of helical magnetohydrodynamic turbulence. J. Fluid Mech. 85, 305.

Roberts, D. A., Goldstein, M. L., Klein, L. W. \& Matthaeus, W. H. $1987 a$ Origin and evolution of fluctuations in the solar wind: Helios observations and Helios-Voyager comparisons. J. Geophys. Res. 92, 12023.

Roberts, D. A., Klein, L. W., Goldstern, M. L. \& Matthaeus, W. H. $1987 b$ The nature and evolution of magnetohydrodynamic fluctuations in the solar wind: Voyager observations. J. Geophys. Res. 92, 11021.

Shebalin, J. V., Matthaeus, W. H. \& Montgomery, D. 1983 Anisotropy in mhd turbulence due to a mean magnetic field. J. Plasma Phys. 29, 525 (referred to herein as SMM).

STRaUSS, H. R. 1976 Nonlinear, three-dimensional magnetohydrodynamics of noncircular tokamaks. Phys. Fluids 19, 134.

Stribling, T. \& Matthaeus, W. H. 1990 Statistical properties of ideal three-dimensional magnetohydrodynamics. Phys. Fluids B 2, 1979.

Stribling, T. \& Matthaeus, W. H. 1991 Relaxation processes in a low order three-dimensional magnetohydrodynamics model. Phys. Fluids B 3, 1848.

Stribling, T., Matthaeus, W. H. \& GHosh, S. 1994 a Nonlinear decay of magnetic helicity in magnetohydrodynamics with a mean magnetic field. J. Geophys. Res. 99, 2567.

Stribling, T., Matthaeus, W. H. \& Oughton, S. $1994 \mathrm{~b}$ Magnetic helicity in magnetohydrodynamic turbulence with a mean magnetic field. Phys. Plasmas (in press).

StRibling, W. T. 1991 Relaxation processes in turbulent magnetohydrodynamic decay. $\mathrm{PhD}$ thesis, University of Delaware.

Ting, A. C., Matthaeus, W. H. \& Montgomery, D. 1986 Turbulent relaxation processes in magnetohydrodynamics. Phys. Fluids 29, 3261.

Zank, G. P. \& Matthaeus, W. H. $1992 a$ The equations of reduced magnetohydrodynamics. J. Plasma Phys. 48, 85.

Zank, G. P. \& Matthaeus, W. H. $1992 b$ Waves and turbulence in the solar wind. J. Geophys. Res. 97, 17189.

ZaNK, G. P. \& Matrhaeus, W. H. 1993 Nearly incompressible fluids II: Magnetohydrodynamics, turbulence, and waves. Phys. Fluids A 5, 257. 